\title{
Multiple Congenital Anomalies in a Patient with Interstitial 6q26 Deletion
}

\author{
Surasak Puvabanditsin Emily Negroponte Peter Jang Amanda Hedges \\ Ramnan Kased Rajeev Mehta \\ Department of Pediatrics, Rutgers Robert Wood Johnson (RWJ) Medical School, New Brunswick, NJ, USA
}

\author{
Keywords \\ Chromosomal anomaly · Meningomyelocele . \\ Microdeletion 6q26 · Spina bifida
}

\begin{abstract}
We report a preterm male neonate presenting with a lumbosacral meningomyelocele, type II Arnold Chiari malformation, hypoplasia of the aortic arch, bicuspid aortic valve, ventricular septal defect, secundum atrial septal defect, multicystic dysplastic kidney, and hydronephrosis. Analysis with whole genome SNP microarray revealed an interstitial deletion of about $237 \mathrm{~kb}$ in chromosome $6 q 26$. Long contiguous stretches of homozygosity (>3 Mb) were seen in 18 chromosomes with a total genomic size of $219 \mathrm{Mb}$. The phenotype seen in our patient has not been reported in association with the genes in the homozygous regions. However, our patient shares many phenotypic features with other reported cases that have shown a deletion in the same region of chromo-
\end{abstract} some 6 .

(c) 2019 S. Karger AG, Basel

Interstitial deletions involving terminal $6 \mathrm{q}(6 \mathrm{q} 26 \mathrm{q}-$ term) are rare chromosomal abnormalities associated with variable phenotypes. Previous case reports characterized the patients with intellectual disability, feeding difficulties, delayed language development and dysprax-

\section{KARGER}

(c) 2019 S. Karger AG, Basel

E-Mail karger@karger.com

www.karger.com/msy ia, sacral dimple, spinal lipoma, seizures, low muscle tone, brain malformations (microcephaly, colpocephaly, absent corpus callosum), cardiac defects, and minor dysmorphic features [Striano et al., 2006; De Cinque et al., 2017; Unique, 2019]. We report a new phenotype in a neonate with a $6 \mathrm{q} 26$ microdeletion.

\section{Case Report}

Our patient is an Indian male neonate born at 36 weeks' gestation to a 25 -year-old primigravida by cesarean section. Apgar scores were 9 and 9 at 1 and 5 min, respectively. Pregnancy was complicated by an abnormal prenatal ultrasound at 20 weeks' gestation, wherein a neural tube defect (NTD) was noted at the lumbosacral (LS) region. Subsequent sonography revealed an additional kyphosis anomaly. Genetic studies had not been performed during pregnancy. The family history was negative for congenital anomalies, and there was no history of in utero exposure to any known teratogens. The parents are first cousins. Physical examination revealed a birth weight of 2,300 g (10th centile), length $43 \mathrm{~cm}$ (5th centile), and a head circumference of $32 \mathrm{~cm}$ (25th centile). Anomalies noted at birth included trigonocephaly, wide anterior fontanelle, wide saggital suture, low-set ears, LS meningomyelocele, kyphosis, hypotonia of both lower extremities without spontaneous movement, and bilateral club feet (Fig. 1). Neurosonogram and cranial computerized tomography showed type II Arnold Chiari malformation, bilateral lateral ventriculomegaly, and third ventriculomegaly (Fig. 2). Echocardiography revealed a small secundum atrial septal defect, multiple anterior apical muscular ventricular septal defects, mild hypoplasia of the aortic isthmus, and bicuspid aortic valve. Renal sonography showed a left multicystic 


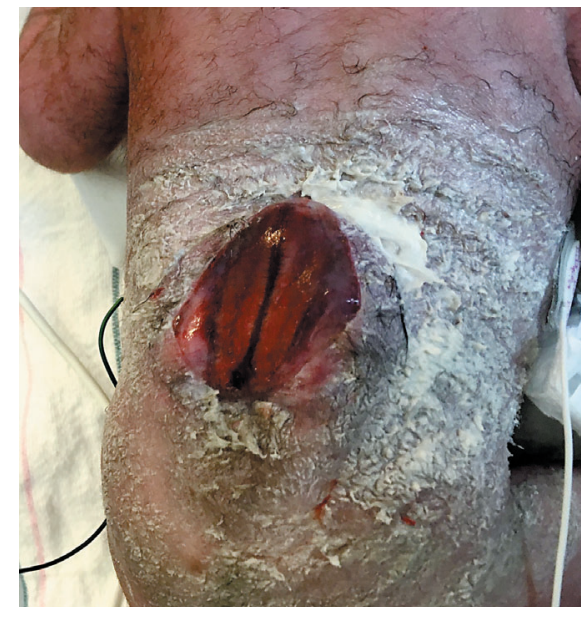

Fig. 1. Lumbosacral myelomeningocele.

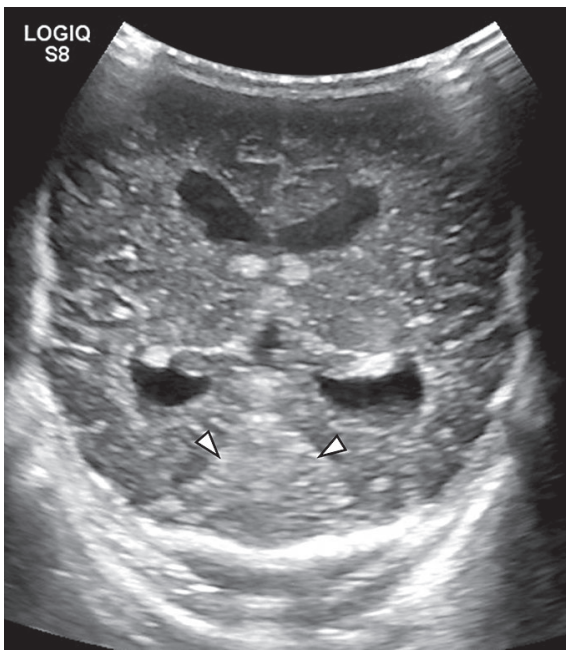

Fig. 2. Neurosonography revealing a hydrocephalus (dilated lateral and third ventricles), small and crowding posterior fossa (arrowheads), and compressed fourth ventricle seen in type II Arnold Chiari malformation.

dysplastic kidney and mild right-sided hydronephrosis (Fig. 3, 4). The patient underwent surgical repair of the myelomeningocele and removal of the 3rd lumbar vertebra to correct the kyphosis. Subsequently, a ventriculoperitoneal shunt was placed on the 11th day of life due to progressive hydrocephalus. The infant remained in the hospital for 21 days because of feeding difficulties.

\section{Materials and Methods}

Whole genome SNP microarray analysis was performed using the SNP oligonucleotide microarray analysis (SOMA) CytoScan HD platform, which uses over 2,696,550 NPCN probes. There was a 237.2-kb deletion in the long arm of chromosome 6, arr [GRCh38]

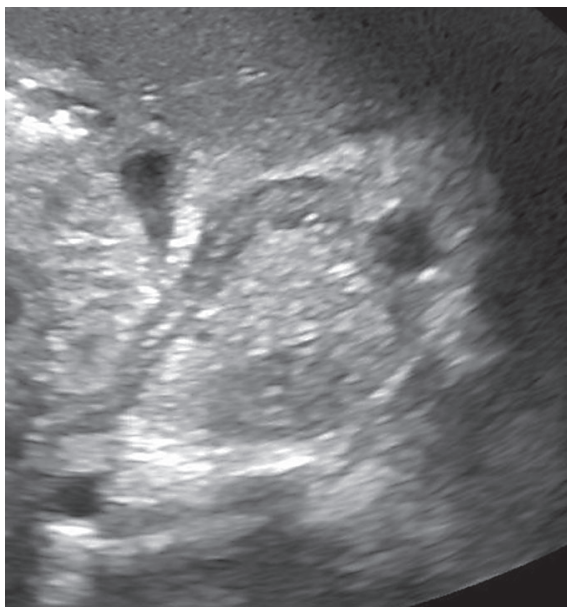

Fig. 3. Renal sonography showing a right multicystic dysplastic kidney.

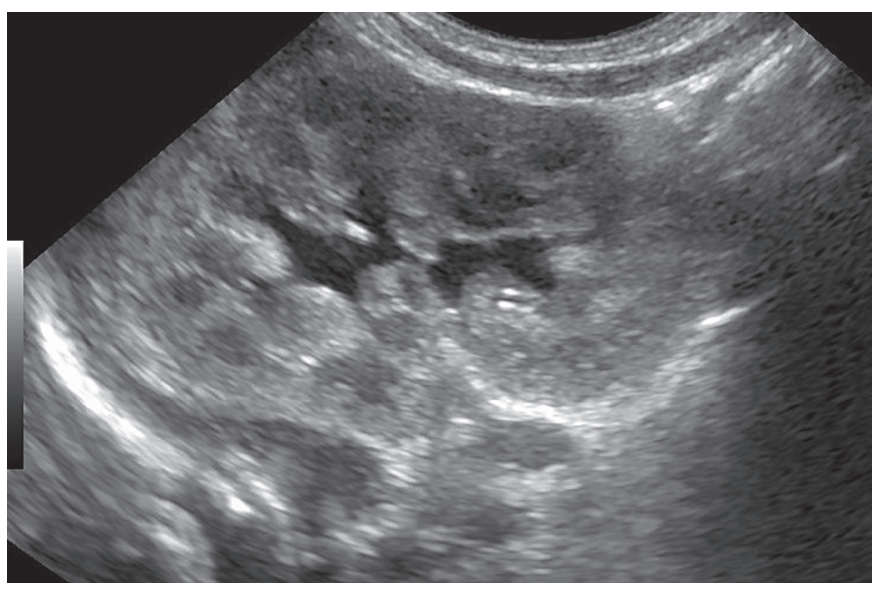

Fig. 4. Renal sonography showing a left hydronephrosis.

6q26(162594940_162832227) $\times 1$ [ISCN 2016]. The SOMA indicated the loss of chromosome 6 from positions 162,594,940$162,832,227$. This deletion region contains a partial deletion of the PARK2 gene (exons 3-4).

Additionally, there were long contiguous stretches of homozygosity $(\mathrm{LCSH})(>3 \mathrm{Mb}$ ) observed throughout the genome, consistent with the presence of a limited gene pool in the isolate populations. The LCSH regions are listed in Table 1.

This finding reflects the increased risk of recessive allele pairing present in that population.

\section{Discussion}

Interstitial deletion in terminal 6q (6q25qter) was first reported in 1975. It is a rare chromosomal abnormality that is associated with a variable phenotype spectrum 
Table 1. Regions of homozygosity

\begin{tabular}{|c|c|c|c|c|}
\hline $\begin{array}{l}\text { Chromo- } \\
\text { some }\end{array}$ & $\begin{array}{l}\text { Genomic coordinates, } \\
\text { hg19 }\end{array}$ & $\begin{array}{l}\text { Length of } \\
\text { ROH, bp }\end{array}$ & $\begin{array}{l}\text { OMIM } \\
\text { genes, } n\end{array}$ & Affected protein-coding genes \\
\hline 2 & $15,702-8,226,542$ & $8,210,841$ & 17 & TPO, PXDN, MYT1L, TRAPPC12, RNASEH1, RPS7, CLK1, SOX11 \\
\hline 3 & $62,613-36,58,712$ & $3,596,100$ & 6 & TRNT1, CRBN \\
\hline 3 & $110,146,639-134,858,117$ & $24,711,479$ & 145 & $\begin{array}{l}\text { ZBTB20, ARHGAP31, TIMMDC1, HGD, ADCY5, ZNF148, SEC61A1, RAB7, GP9, } \\
\text { CNBP, RHO, MRPL3, BFSP2 }\end{array}$ \\
\hline 4 & $167,700,119-170,778,068$ & $3,077,950$ & 6 & $P A L L D, N E K 1$ \\
\hline 5 & $60,888,201-66,502,710$ & $5,614,510$ & 15 & KIF2A, CWC27 \\
\hline 5 & $149,245,753-157,588,362$ & $8,342,610$ & 55 & PDE6A, NDST1, SPARC, SGCD, ITK, CYFIP2 \\
\hline 6 & $147,341,369-150,802,064$ & $3,460,696$ & 19 & SASH1, TAB2, IYD \\
\hline 6 & $138,811,327-143,512,674$ & $4,701,348$ & 4 & REPS1, ADGRG6, HIVEP2 \\
\hline 7 & $22,145,083-29,819,335$ & $7,674,253$ & 47 & DFNA5, IL6, GPNMB, SNX10 \\
\hline 7 & $43,125,192-55,705,566$ & $12,580,375$ & 50 & BLVRA, PGAM2, AEBP1, GCK, CAMK2B, OGDH, PKD1L1, AADC \\
\hline 7 & $83,261,522-96,946,490$ & $13,684,969$ & 57 & $\begin{array}{l}\text { SEMA3A, ADAM22, CFAP69, PEX1, CDK6, SAMD9, COL1A2, SGCE, PON1, } \\
\text { SEM1 }\end{array}$ \\
\hline 9 & $137,786,802-141,025,328$ & $3,238,527$ & 59 & PMPCA, INPP5E, AGPAT2, MAN1B1, EHMT1 \\
\hline 9 & $79,438,031-85,535,897$ & $6,097,867$ & 10 & GNAQ, PSAT1, CEP78 \\
\hline 10 & $66,309,197-73,351,845$ & $7,042,649$ & 46 & $\begin{array}{l}\text { CTNNA3, MYPN, KBP, HK1, NEUROG3, COL13A1, PRF1, SGPL1, PCBD1, } \\
\text { SLC29A3, PSAP }\end{array}$ \\
\hline 11 & $98,889,146-107,624,198$ & $8,735,053$ & 40 & CFAP300, YAP1, MMP20, MMP1, MMP13, DYNC2H1, ACAT1 \\
\hline 12 & $11,969,598-27,856,426$ & $15,886,829$ & 75 & CDKN1B, GUCY2C, ART4, MGP, PDE6H, PDE3A, SLCO1B1, PYROXD1, GYS2 \\
\hline 18 & $2,096,400-10,202,532$ & $8,106,133$ & 25 & SMCHD1, LPIN2, TGIF, NDUFV2 \\
\hline 19 & $28,273,327-31,814,167$ & $3,540,841$ & 0 & \\
\hline 19 & $260,911-24,505,637$ & $24,244,727$ & 451 & $\begin{array}{l}\text { BSG, ELANE, CFD, GPX4, ATP5F1D, NDUFS7, TCF3, ADAT3, AP3D1, PIP5K1C, } \\
\text { MAP2K2, FUT6, ARHGEF18, PNPLA6, ANGPTL4, DNMT1, FDX2, DNM2, } \\
\text { KANK2, ACP5, RNASEH2A, GCDH, CALR, TRMT1, CACNA1A, CC2D1A, } \\
\text { PRKACA, TECR, CASP14, KIAA1283, JAK3, PIK3R2, CRLF1, CRTC1, COMP, } \\
\text { NDUFA13 }\end{array}$ \\
\hline 20 & $45,905,751-49,496,121$ & $3,590,371$ & 23 & ARFGEF2, PTGIS, PTPN1, DPM1 \\
\hline 21 & $39,311,419-43,587,562$ & $4,276,144$ & 35 & RIPK4, TMPRSS3, WDR4, CBS, CRYAA \\
\hline
\end{tabular}

$\mathrm{ROH}$, region of homozygosity. ROH available at http://firefly.ccs.miami.edu/cgi-bin/ROH/ROH_analysis_tool_for_trial.cgi. 
[Bertini et al., 2006; Lee at al., 2011]. The 6q distal deletion is characterized by a highly variable clinical manifestation that does not depend on extension of the chromosomal abnormalities [Stevenson et al., 2004; Bertini et al., 2006; Lee et al., 2011]. This is known to also happen in other contiguous gene deletion syndromes because any copy number variation $(\mathrm{CNV})$ can affect the expression level of genes situated in the vicinity of the deleted or duplicated region, thus modulating the clinical consequences of haploinsufficiency. This "position effect" can explain the complexity and heterogeneity of the phenotype associated with terminal 6q deletions [Stranger et al., 2007].

The reported clinical features of the deletion include brain abnormalities (agenesis/hypoplasia of corpus callosum, microcephaly, nodular heterotopy), spinal anomalies (LS scoliosis, tethered cord, spinal lipoma/dimple), intellectual disabilities, seizures, hypotonia, congenital heart defects (bicuspid aortic valve, atrial septal defect, ventricular septal defect, dextrocardia), and genitourinary abnormalities (hydronephrosis, cryptorchidism) [Bertini et al., 2006; Lee at al., 2011; De Cinque et al., 2017; Unique, 2019].

Both genetic and nongenetic factors contribute to NTDs. In the 1960s, British prevalence surveys that were based on the relative proportions of individuals affected among siblings of index cases estimated heritability (the genetic component of risk) at $60-70 \%$. Most of the NTD cases are nonsyndromic and exhibit a sporadic pattern of occurrence. Only 10\% are syndromic and occur in chromosomal disorders such as trisomy 13 or 18 . The multifactorial causation model for nonsyndromic NTDs suggests the involvement of multiple genes and nongenetic factors [Copp et al., 2015]. The best-known nongenetic factor influencing NTD risk is diminished folate status. Some nutrients and nutrition-related factors such as alcohol, smoking, caffeine, and valproic acid are also associated with NTDs. Other factors that have been linked with NTDs include hyperthermia, a low socioeconomic status, and psychosocial stress. Population studies have reported a relationship with maternal obesity. Suggested mechanisms include aberrant glucose control, oxidative stress, and metabolic syndrome. Obesity-associated risks are stronger for spina bifida than for anencephaly. Infrequently, exposure to a variety of environmental factors such as air pollution, nitrates, organic solvents, pesticides, and polycyclic aromatic hydrocarbon have been linked with NTDs. Because the associated magnitude in risk for these factors is not very large, they cannot explain a substantive proportion of the population burden of NTDs [Copp et al., 2015].

Interstitial 6q26 Deletion and Congenital Anomalies
Long contiguous regions of homozygosity (ROHs) may arise in situations such as parental relatedness (or consanguinity) and chromosomal recombination or rearrangements [Wierenga et al., 2013]. The chromosomal distribution and cumulative extent may be indicative of the background ancestral homozygosity, uniparental disomy (UPD) or parental consanguinity [Wang et al., 2015]. The criterion for reporting UPD is the finding of a single chromosome with LCSH greater than $15 \mathrm{Mb}$. Finding of multiple chromosomes with LCSH greater than 10 $\mathrm{Mb}$ is used for the reporting of identity by descent (consanguinity). Although short ROHs may also carry valuable information, some laboratories report only relatively long ROHs (longer than 8 or $10 \mathrm{Mb}$ ) [Deak et al., 2005a].

Our patient has spinal abnormalities (LS meningomyelocele), genitourinary abnormalities (cystic kidney and hydronephrosis), and cardiac defects (atrial/ventricular septal defect). These anomalies are similar to those reported in the literature. The parents had emigrated from India and are related. The mother does not smoke, drink alcohol, or take anticonvulsant medication. There was no history of exposure to any known teratogens. Genomic microarray of our patient showed a 237.2-kb deletion of $6 \mathrm{q} 26$. The candidate genes associated with LS meningomyelocele are NCAM1, ALDH1A2, CYP26A1, CYP26B1, $C R A B P 1$, and CRABP2 [Deak et al., 2005b]. We did not find these genes in our patient's ROHs. The SNP array evaluation tool functions optimally when the ROHtotal is between 50 and $400 \mathrm{Mb}$. Our patient's ROHtotal was about $254 \mathrm{Mb}$. The size of the homozygous segment associated with recessive disease was $26 \mathrm{Mb}$ (range 5-70 $\mathrm{Mb})$.

We entered our patient's data into the search page of the SNP array evaluation tool (Genomic Oligoarray and SNP array evaluation tool v3.0) [Wang et al., 2015] but could not arrive at a diagnosis. Alongside the background of consanguinity, such a negative finding adds to the suspicion that the disorder may not have been documented before, or more likely, the causative locus has not yet been mapped.

To the best of our knowledge, very few patients with isolated $6 \mathrm{q} 26$ region deletions (identified by array CGH) have been reported, and none were diagnosed with the phenotype seen in our patient. Our case revealed distinct phenotypic findings: LS myelomeningocele, hypoplasia of the aortic isthmus, and a multicystic dysplastic kidney.

In summary, we report a case of a neonate with a $6 \mathrm{q} 26$ microdeletion that is associated with new phenotypic findings. Our report could help expand the phenotype of isolated $6 \mathrm{q}$ terminal deletions. 


\section{Acknowledgment}

We thank Sylvia Sutton-Thorpe, Chrystal Puvabanditsin, and Christina Puvabanditsin for their support and preparing the manuscript.

\section{Statement of Ethics}

The authors have no ethical conflicts to declare.

\section{Disclosure Statement}

The authors have no conflicts of interest to disclose.

\section{References}

Bertini V, De Vito G, Costa R, Simi P, Valetto A: Isolated $6 \mathrm{q}$ terminal deletions: an emerging new syndrome. Am J Med Genet A 140:74-81 (2006).

Copp AJ, Adzick NS, Chitty LS, Fletcher JM, Holmbeck GN, Shaw GM: Spina bifida. Nat Rev Dis Primers 1:15007 (2015).

Deak KL, Dickerson ME, Linney E, Enterline DS, George TM, et al: Analysis of ALDH1A2, CYP26A1, CYP26B1, CRABP1, and CRABP2 in human neural tube defects suggests a possible association with alleles in $A L D H 1 A 2$. Birth Defects Res A Clin Mol Teratol 73:868875 (2005a).

Deak KL, Boyles AL, Etchevers HC, Melvin EC, Siegel DG, et al: SNPs in the neural cell adhesion molecule 1 gene (NCAM1) may be associated with human neural tube defects. Hum Genet 117:133-142 (2005b).
De Cinque M, Palumbo O, Mazzucco E, Simone A, Palumbo P, et al: Developmental coordination disorder in a patient with mental disability and a phenotype carrying terminal 6q26qter deletion. Front Genet 8:206 (2017).

ISCN 2016: An International System for Human Cytogenomic Nomenclature; McGowan-Jordan J, Simons A, Schmid M (eds). Cytogenet Genome Res 149:1-140 (2016).

Lee JY, Cho YH, Hallford G: Delineation of subtelomeric deletion of the long arm of chromosome 6. Ann Human Genet 75:755-764 (2011).

Stevenson DA, Brothman AR, Carey JC, Chen Z, Dent $\mathrm{KM}$, et al: $6 \mathrm{q}$ subtelomeric deletion: is there a recognizable syndrome? Clin Dysmorphol 13:103-106 (2004).

Stranger BE, Forrest MS, Dunning M, Ingle CE, Beazley C, et al: Relative impact of nucleotide and copy number variation on gene expression phenotypes. Science 315:848-853 (2007).
Striano P, Malacarne M, Cavani S, Pierluigi M, Rinaldi R, et al: Clinical phenotype and molecular characterization of $6 \mathrm{q}$ terminal deletion syndrome: five new cases. Am J Med Genet A 140:1944-1949 (2006).

Unique 2019: Understanding rare chromosome and gene disorders https://www.rarechromo. org/media/information/chromosome $\% 20$ \%206/6q\%20deletions\%20from\%206q26\%20 and\%206q27\%20FTNP.pdf.

Wang JC, Ross L, Mahon LW, Owen R, Hemmat $\mathrm{M}$, et al: Regions of homozygosity identified by oligonucleotide SNP arrays: evaluating the incidence and clinical utility. Eur J Hum Genet 23:663-671 (2015).

Wierenga KJ, Jiang Z, Yang AC, Mulvihill JJ, Tsinoremas NF, et al: A clinical evaluation tool for SNP arrays, especially for autosomal recessive conditions in offspring of consanguineous parents. Genet Med 15:354-360 (2013). 